COMMENT. Children with rolandic epilepsy are predisposed to impairments of attention that resolve with remission of EEG centrotemporal spikes. Attention impairment in RE is correlated not with seizures but with EEG epileptiform discharges. Children with RE should be monitored for attention, language, and learning disorders. Rolandic spikes may aggravate the course of ADHD and predispose to increased impulsivity (Holtmann M et al. Brain Dev 2006;28:633-640).

\title{
ANXIETY AND DEPRESSION WITH FIRST SEIZURE
}

Anxiety and depressive signs were investigated in 22 children (mean age, 12+/-2.7 years SD) with a first unprovoked afebrile seizure, in a study at Alberta Children's Hospital, University of Calgary, Canada. Compared with published norms for a Child Anxiety Scale and Depression Inventory, patients reported significantly higher scores for total anxiety, worry/oversensitivity, and social concerns/concentration. Compared with a medical cohort, anxiety symptoms were similar. Children with a first seizure had greater interpersonal problems, ineffectiveness, and negative self-esteem than published norms. Compared to controls, they had increased negative mood, ineffectiveness, anhedonia, and negative selfesteem. Anxiety may be related to illness or hospital experience, and depressive symptoms may be comorbid at time of seizure. (Loney JC, Wirrell EC, Sherman EMS, Hamiwka LD. Anxiety and depressive symptoms in children presenting with a first seizure. Pediatr Neurol Oct 2008;39:236-240). (Respond: Dr Hamiwka, Division of Pediatric Neurology, Alberta Children's Hospital, 2888 Shaganappi Trail NW, Calgary, Alberta T3B 6A8, Canada. Email: ihamiwka(a;mac.com).

COMMENT. Children presenting with a first seizure may be at increased risk of anxiety and depressive symptoms.

\section{DEMYELINATING DISORDERS}

\section{RISK FACTORS FOR MULTIPLE SCLEROSIS AFTER FIRST ATT ACK OF INFLAMMATORY CNS DEMYELINATION}

Clinical, radiologic, or CSF factors predicting development of multiple sclerosis (MS) after a first inflammatory demyelinating attack were identified in 117 children $(56 \%<10$ years, $34 \%<6$ years) participating in a nationwide retrospective multicenter study in the Netherlands. A second MS-defining attack occurred in $43 \%$ of 54 children who presented with a monofocal clinically isolated syndrome (CIS), compared to $21 \%$ of 63 patients with a polyfocal CIS $(\mathrm{p}<0.006)$. Lesions considered typical of ADEM (basal ganglia and thalamic lesions and lesions $>2 \mathrm{~cm}$ on MRI) occurred during PCIS, with or without encephalopathy. Children with PCIS had a preceding infection in $50 \%$, fever in $42 \%$, and seizures in $28 \%$; they were not at greater risk of developing MS. Children with PCIS without encephalopathy progressed to MS more frequently than those with encephalopathy (ADEM). Risk factors for development of MS included elevated IgG index, oligoclonal CSF bands, 3 Barkhof MRI criteria ( $>9$ lesions T2, infratentorial, juxtacortical, and $>3$ perivascular lesions), and KIDMUS supplemental MRI criteria (perpendicular to corpus callosum, thalamic/basal 